\title{
Accurate predictions of the EPR parameters in planar cobalt(II) complexes by hybrid density functional theory
}

\author{
Mohamed Zbiri \\ Department of Chemistry, University of Fribourg, 9 Chemin du Musée, Pérolles, 1700 Fribourg, Switzerland
}

\begin{abstract}
The hybrid density functional theory is applied to calculate the electron paramagnetic resonance parameters, i.e, the g- and A-tensors of some planar Cobalt(II) complexes with a $C_{2 v}$ symmetry. Calculations were done for four systems: Co(acacen), Co(tacacen), Co(seacacen) and $\mathrm{Co}\left(\mathrm{sacsac}_{2}\right.$. The following hybrid functionals were employed: B3LYP, B3PW91, mPW1PW91 and PBE0. The expected large deviation of the g- and A-tensors is well reproduced, and is in very good agreement with the experimental observations. Comparative study shows that the $P B E O$ hybrid model yields the best agreement with experimental data.
\end{abstract}

Keywords: Hybrid DFT; EPR parameters; Cobalt complexes; Planar symmetry

\section{Introduction}

Electron paramagnetic resonance (EPR) is a powerful experimental technique allowing to extract details of the electronic structure of complexes with unpaired electrons [1]. EPR can be used to map the distribution of an unpaired electron in a molecule and, thus obtain informations to which extent the electrons are delocalized over the ligands. It can also provide some information about the energy levels of complexes. Extraction of these informations from experimental spectra is however not always straightforward, quantum-chemical calculations are therefore needed [2]. Calculations of the g- and A-tensors within traditional ab initio approaches require sophisticated treatment of the electron correlation and large basis sets, which is computationally demanding [3]. These reasons make DFT a suitable tool for such a calculations $[4,5]$. However, standard XC functionals (GGAs) underestimate the magnetic resonance parameters in transition metal complexes [6,7]. For the same systems, the core-

E-mail address: mohamed.zbiri@unifr.ch. shell spin polarization effect are also underestimated [8], this can be improved by using the hybrid density functionals (HDFT).

In a previous work [5], we have investigated the anisotropic behavior of the EPR parameters; i.e. the g- and the A-tensors, of the Co(acacen) complex using pure density functionals (pure GGAs) based on a second-order perturbation theory combined with the zeroth-order regular approximation (ZORA) [9,10]. As it was reported [5], the results were in a rather good agreement with the experimental data. However, a predefined amount of exact Hartree-Fock (HF) exchange can be added to the pure density functionals which leads to the well known hybrid functionals. Arbuznikov and coworkers [11] proposed a new scheme and first implementation to calculate the electronic g- and A-tensors, for a variety of systems, especially transition metal complexes (TM). They used meta-GGA $[12,13]$ exchange-correlation functionals, wherein both Laplacian and kinetic-energy-density are taken into account, and statistically average orbital model exchange-correlation potentials [14] (SAOP). Munzarova and Kaupp [15] presented a critical study of the density functional approach for calculation of the A-tensor in transition metal com- 
plexes, they have compared some combinations of exchange and correlation potentials, using pure and hybrid functionals.

The reason behind the design and the use of new hybrid and meta functionals to predict magnetic resonance parameters, is that the standard GGA exchange-correlation functionals underestimate these parameters in transition metal complexes $[6,7]$. For the same systems, the core-shell spin polarization effect are also underestimated [8], this can be improved by using hybrid functionals [16]. Recently, the hybrid models were used efficiently to elucidate the magnetic behavior of a set of a newly synthesized azido-bridged binuclear complexes by calculating their magnetic exchange coupling constants [17]. Within this spirit, the aim of the present communication is to show the ability of the HDFT methods to predict and evaluate accurately other type of magnetic properties of transition metal systems; i.e. the g- and A-tensors. Four hybrid models are used and their performance will be discussed via comparative study in Section 4.

Cobalt(II) complexes are interesting and suitable systems for such investigation, $\mathrm{Co}(\mathrm{II})$ forms low-spin $\mathrm{d}^{7}$-complexes with tetradentate $S$ chiff bases [18] which leads nearly to a $C_{2 v}$-symmetry. As explained in a previous study [5], the main consequence of that planar coordination is related to the electronic energy levels of the five d-orbitals of the metal. The typical splitting for square planar coordination, i.e considering the highest occupied $\pi\left(\mathrm{a}_{2}\right)(\mathrm{HOMO})$ and the lowest unoccupied $\pi^{*}\left(b_{1}\right)$ (LUMO) molecular orbitals, are due to the fact that $3 \mathrm{~d}$-orbitals with the same symmetry become destabilized or stabilized by mean of $\pi$-donation or $\pi$-back donation, as a consequence of this anisotropic $\pi$-bonding is the large in-planar anisotropy of the main values of the g- and A-tensors.

\section{The hybrid functionals}

The hybrid DFT/HF methods are rooted in the adiabatic connection formula/concept $[19,20]$. The best way to build "accurate" HDFs is to use this concept. In this approach, an adiabatic parameter $\lambda$ is introduced to tune the electron-electron interaction for a given system taken a sum of fictitious systems all with density $\rho$ and different e-e interaction

$E_{\mathrm{xc}}=\int_{0}^{1} E_{\mathrm{xc}, \lambda} \mathrm{d} \lambda$

$\lambda=0$ corresponds to non-interacting electrons,

$E_{\mathrm{xc}, \lambda=0}=E_{\mathrm{X}}=F_{\mathrm{HF}}$

$\lambda=1$ corresponds to full interacting electrons,

$E_{\mathrm{xc}, \lambda=1}=E_{\mathrm{xc}}+T-T_{\mathrm{S}}$

In Eq. (1), the adiabatic parameter $\lambda$ has two extreme values 0 and 1 corresponding to Eqs. (2) and (3). But the difficulty arises when $\lambda$ varies between these two values. However, one can overcome this problem by writing the
$\mathrm{XC}$ energy as a linear expansion with respect to $\lambda$. Starting from the adiabatic connection formula, several hybrid schemes have been proposed, most of them include three empirical parameters to determine the mixing of HF and DFT exchange, and correlation. The B3LYP [26] hybrid scheme is an example of hybrid functional determined by semiempirical approach

$E_{\mathrm{xc}}^{\mathrm{B} 3 \mathrm{LYP}}=(1-a) E_{\mathrm{xc}}^{\mathrm{LSD}}+a E_{\mathrm{xc}}^{\lambda=0}+b E_{\mathrm{X}}^{B 88}+c E_{\mathrm{C}}^{\mathrm{LYP}}+(1-c) E_{\mathrm{C}}^{\mathrm{LSD}}$

where $a=0.20, b=0.72$, and $c=0.81$, are the semiempirical coefficients determined by an appropriate fit to experimental data, $E_{\mathrm{xc}}^{\lambda=0}$ is the exact exchange energy (tuned by a), $E_{\mathrm{X}}^{\mathrm{B}}$ is Becke's 1988 gradient correction (to the LSDA) for exchange [21], and $E_{\mathrm{C}}^{\mathrm{LYP}}$ is the Lee, Yang, and Parr gradient correction [25]. The second strategy to build a new hybrid model is the non-parametric way. The PBE0 $[28,29]$ hybrid functional is a good example of a functional without adjustable parameters. The idea is to use the pure GGA PBE [30,31], and to add $25 \%$ of the HF exchange to it based on purely theoretical and physical considerations. In this case, the hybrid functional is written with respect to $\lambda$ as follows:

$E_{\mathrm{xc}, \mathrm{HDF}}^{\lambda}(n)=E_{\mathrm{xc}, \mathrm{DF}}^{\lambda}+\left(E_{\mathrm{X}, \mathrm{HF}}-E_{\mathrm{X}, \mathrm{DF}}\right)(1-\lambda)^{n-1}$

where $n \geqslant 1$ is an integer to be determined, it controls how rapidly the correction to DF vanishes as $\lambda \rightarrow 1$. Then

$E_{\mathrm{xc}, \mathrm{HDF}}=\int_{0}^{1} \mathrm{~d} \lambda E_{\mathrm{xc}, \mathrm{HDF}}^{\lambda}=E_{\mathrm{xc}, \mathrm{DF}}+\frac{1}{n}\left(E_{\mathrm{X}, \mathrm{HF}}-E_{\mathrm{X}, \mathrm{DF}}\right)$

The optimum integer $n$ should be the lowest order [28] of perturbation theory which provides a realistic description of the shape or $\lambda$-dependence of the exact $E_{\mathrm{xc}}^{\lambda}$. An ideal hybrid functional would be sophisticated enough to optimize $\mathrm{n}$ for each system and property, but the accuracy of MP4 (fourth-order Møller-Plesset perturbation theory) [32] for most molecules suggests $n=4$ as the best single choice,

$E_{\mathrm{xc}}^{\mathrm{HDF}}(n=4)=E_{\mathrm{xc}}^{\mathrm{DF}}+\frac{1}{4}\left(E_{\mathrm{X}}^{\mathrm{HF}}+E_{\mathrm{X}}^{\mathrm{DF}}\right)$

As explained above, the so-called PBE0 model is a variant obtained inserting the PBE (DF or GGA) in Eq. (7),

$E_{\mathrm{xc}}^{\mathrm{PBE} 0}=E_{\mathrm{xc}}^{\mathrm{PBE}}+0.25\left(E_{\mathrm{X}}^{\mathrm{HF}}+E_{\mathrm{X}}^{\mathrm{PBE}}\right)$

this functional does not contain any fitted parameter to experimental data, and that a predefined amount of HF exchange is added self-consistently to DFT contribution, $P B E 0$ model can be considered a true non-empirical DFT approach. This model generally provides results which are of similar accuracy as those obtained with empirical functionals $[40,41]$.

\section{Computational details}

All calculations were performed using the GAUSSIAN program [33] (version 03), with the following exchange- 

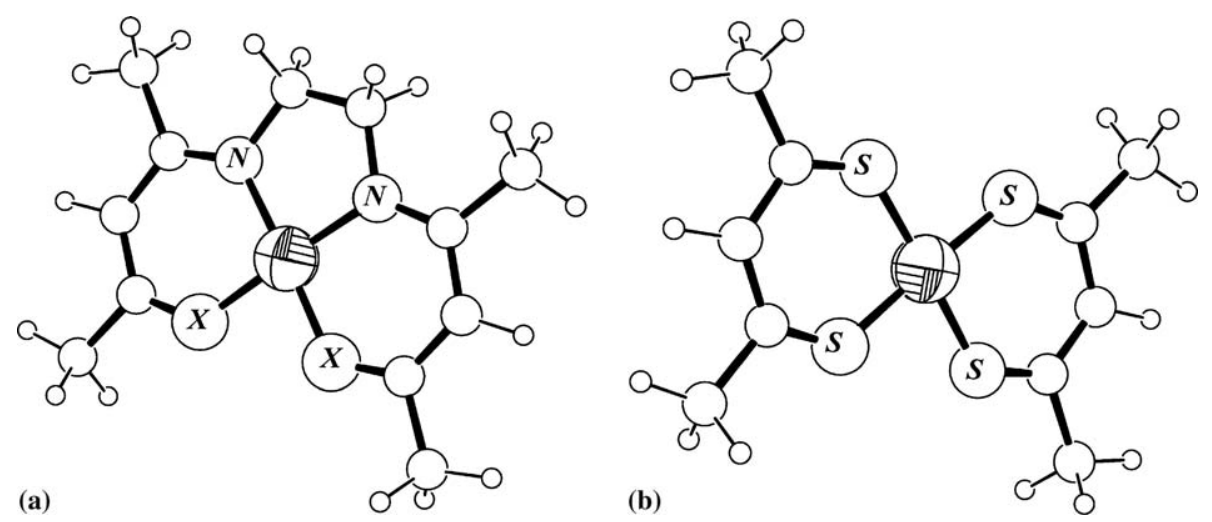

Fig. 1. The Co(II) complexes: (a) X stands for oxygen (O), sulfur (S) and selenium (Se) corresponding to co(acacen), co(tacacen) and co(seacacen), respectively. (b) Represents the fourth complex $\operatorname{co}(\operatorname{sacsac})_{2}$.

correlation hybrid functionals, B3LYP [34-36], B3PW91 [34,37,38], mPW1PW91 [39], and PBE0 [40]. To avoid gauge dependence of the $\mathrm{g}$-values upon the origin chosen for the magnetic field if one uses a finite basis set, the gauge including atomic orbitals (GIAO) $[42,43]$ method was used. The experimental geometries based on X-ray crystallographic data determined by Cariati and coworkers [44] were adopted. Calculations were done for four $\mathrm{Co}(\mathrm{II}) \mathrm{com}$ plexes that are shown in Fig. 1. Since the deviation from $C_{2 v}$ symmetry is not significant, this symmetry is imposed in all the calculations. The molecules belong to the point group $C_{2 v}(x)$, with $x$ as the principal symmetry axis instead of the more conventional $z$-axis. By convention, such an orientation has always been used to discuss this type of complexes [5]. All-electron basis sets were used for Co, with $(15 \mathrm{~s} 11 \mathrm{p} 6 \mathrm{~d}) /[9 \mathrm{~s} 7 \mathrm{p} 4 \mathrm{~d}]$ sets designed previously for the hyperfine calculations [15]. The EPR-III basis set, formed following the prescriptions given in Ref. [45], has been used for O, $\mathrm{N}, \mathrm{C}$ and $\mathrm{H}$. The 6-311++ $\mathrm{G}^{* *}$ Gaussian functions [46-48] were adopted for Se and $\mathrm{S}$. The experimental values [18] depend strongly upon the host lattice and the temperature. Thus, a range of values is available. In order to make comparisons with theoretical predictions, only experimental studies in single crystal phase and with nickel as host lattice are considered.

\section{Results and discussion}

\subsection{The g-tensor}

For the current study, the g-tensor is calculated as a correction [49-52], $\Delta \mathbf{g}$, to the free electron values $\mathbf{g}_{\mathrm{e}}$,

$\mathbf{g}=\mathbf{g}_{\mathrm{e}} 1+\Delta \mathbf{g}$

with $\mathbf{g}_{\mathrm{e}}=2.0023193$, and $\Delta \mathbf{g}$ is the correction that represents contributions of three GIAO nuclear magnetic shielding terms:

$\Delta \mathbf{g}=\mathbf{g}_{\mathrm{RMC}}+\mathbf{g}_{\mathrm{DC}}+\mathbf{g}_{\mathrm{OZ}}+\mathbf{g}_{\mathrm{SOC}}$

where $\mathbf{g}_{\mathrm{RMC}}, \mathbf{g}_{\mathrm{DC}}, \mathbf{g}_{\mathrm{OZ}}$ and $\mathbf{g}_{\mathrm{SOC}}$ are relativistic mass correction, diamagnetic correction, orbital Zeeman and spin-orbit coupling [53] contribution, respectively. The calculated $\mathbf{g}$-values of all the complexes using the proposed hybrid methods are summarized in Table 1. The corresponding experimental data is also given for the sake of comparison. Good agreement is found between the calculated and experimental values. The experimental data exhibits a monotonic behavior of the $\mathbf{g}_{z z}$ components for the four complexes. The $\mathbf{g}_{z z}$ values decrease from 2.00 to 1.90 in the following order: $\quad c$ (acacen) $\rightarrow$ co(tacacen $) \rightarrow \operatorname{co}($ seacacen $) \rightarrow \operatorname{co}(\text { (sacsac })_{2}$. For the $\mathbf{g}_{y y}$ component, this trend is only observed for three complexes; i.e. $\operatorname{co}($ tacacen $) \rightarrow \operatorname{co}($ seacacen $) \rightarrow \operatorname{co}(\text { sacsac })_{2}$. This is different for the $\mathbf{g}_{x x}$ values. Indeed, the variation of the $\mathbf{g}_{x x}$ components do not occur monotonically with respect to the change of the ligands forming the first coordination sphere of the TM. Theoretically, these trends are well reproduced by all the hybrid functionals. It is clearly shown that the

Table 1

The calculated and the observed g-tensor values for the cobalt complexes

\begin{tabular}{llllll}
\hline & & Co(acacen) & Co(tacacen) & Co(seacacen) & Co(sacsac) \\
\hline B3LYP & $\mathbf{g}_{x x}$ & 2.85 & 2.83 & 2.93 & 2.91 \\
& $\mathbf{g}_{y y}$ & 1.89 & 1.92 & 1.91 & 1.90 \\
& $\mathbf{g}_{z z}$ & 1.96 & 1.92 & 1.91 & 1.90 \\
& $\mathbf{g}_{i s o}$ & 2.23 & 2.22 & 2.25 & 2.24 \\
B3PW91 & $\mathbf{g}_{x x}$ & 2.87 & 2.85 & 2.95 & 2.92 \\
& $\mathbf{g}_{y y}$ & 1.89 & 1.92 & 1.95 & 1.94 \\
& $\mathbf{g}_{z z}$ & 1.96 & 1.93 & 1.92 & 1.94 \\
& $\mathbf{g}_{i s o}$ & 2.24 & 2.23 & 2.27 & 2.27 \\
mPW1PW91 & $\mathbf{g}_{x x}$ & 3.13 & 3.01 & 3.18 & 3.17 \\
& $\mathbf{g}_{y y}$ & 1.89 & 1.95 & 1.91 & 1.90 \\
& $\mathbf{g}_{z z}$ & 2.00 & 1.96 & 1.93 & 1.93 \\
& $\mathbf{g}_{i s o}$ & 2.34 & 2.31 & 2.34 & 2.33 \\
PBE0 & $\mathbf{g}_{x x}$ & 3.22 & 3.05 & 3.27 & 3.23 \\
& $\mathbf{g}_{y y}$ & 1.89 & 1.96 & 1.94 & 1.92 \\
& $\mathbf{g}_{z z}$ & 2.01 & 1.96 & 1.94 & 1.93 \\
& $\mathbf{g}_{i s o}$ & 2.37 & 2.32 & 2.38 & 2.36 \\
Exp [18] & $\mathbf{g}_{x x}$ & 3.26 & 3.05 & 3.33 & 3.28 \\
& $\mathbf{g}_{y y}$ & 1.88 & 1.98 & 1.93 & 1.90 \\
& $\mathbf{g}_{z z}$ & 2.00 & 1.95 & 1.93 & 1.90 \\
& $\mathbf{g}_{i s o}$ & 2.38 & 2.33 & 2.40 & 2.36 \\
\hline & & & & &
\end{tabular}


PBE0 and mPWPW91 hybrid schemes yields the best estimation of the g-values. The B3LYP and B3PW91 functionals perform equally leading to results that are less accurate than those obtained with PBE0 and mPW1PW91. The $\mathbf{g}_{x x}$ components increase significantly when going from B3LYP to $\mathrm{PBE}$, which proves that there is an influence of the $\mathrm{XC}$ potentials on the description of the anisotropic behavior of the magnetic resonance parameters of TM systems. This is similar to the $\mathbf{g}_{z z}$ component. On the other hand, the same correlation relation is not observed for the $\mathbf{g}_{y y}$ components. But from a quantitative point of view, only the mPW1PW91 and PBE0 hybrid DFs lead to results that are in excellent agreement with the experimental data. In order to complete the comparison and to make a quantitative estimation of the large deviation of the $\mathbf{g}$-values from $\mathbf{g}_{\mathrm{e}}$, the isotropic values of both the calculated and the experimental $\mathbf{g}$-tensors are compared,

$\mathbf{g}_{\text {iso }}=\frac{\mathbf{g}_{x x}+\mathbf{g}_{y y}+\mathbf{g}_{z z}}{3}$

As shown in Fig. 2 (numerical values are also available in Table 1). The PBE0 yields the most accurate predictions of the anisotropy of the g-tensor. Fig. 3 shows the highest occupied (HOMO), lowest unoccupied (LUMO) molecular orbitals, and their separation $\delta$ (the HOMO-LUMO gap). Based on these results, B3LYP and B3PW91 perform

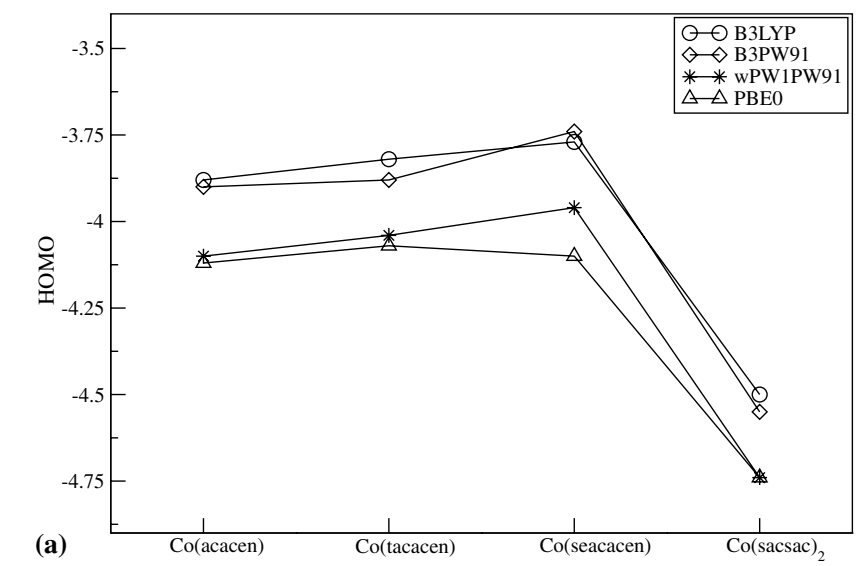

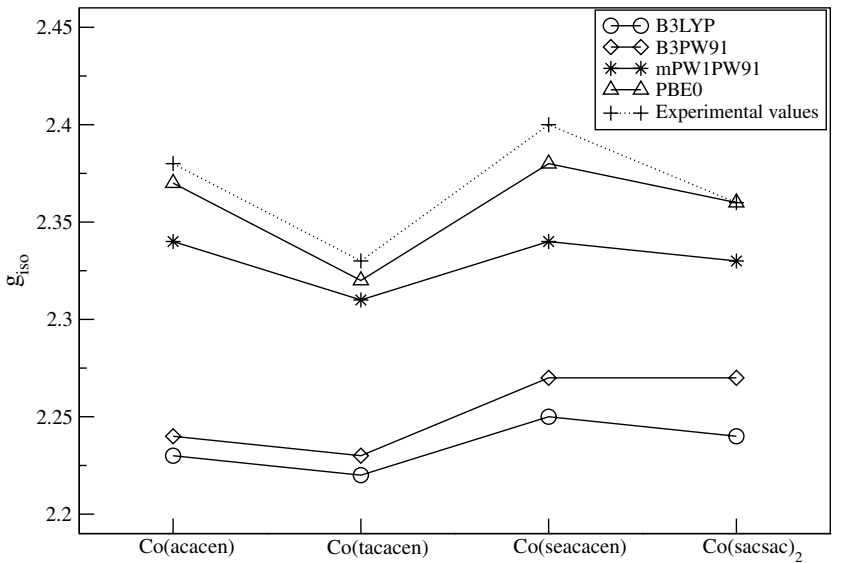

Fig. 2. The theoretical and the experimental [18] isotropic g-values, $\mathbf{g}_{\text {iso }}=\frac{\mathbf{g}_{x x}+\mathbf{g}_{y y}+\mathbf{g}_{z z}}{3}$.

equally to predict the orbital energy levels. The mPW1PW91 and PBE0 hybrid models have also the same performance, and they predict more stabilized HOMO energies and highest values of LUMO levels. Consequently, the HOMO-LUMO gap $\delta$, is much larger when the mPW1PW91 and PBE0 functionals are adopted. This is related to the accuracy of the calculated g-values using these models. As stated in Section 1, the typical splitting of the

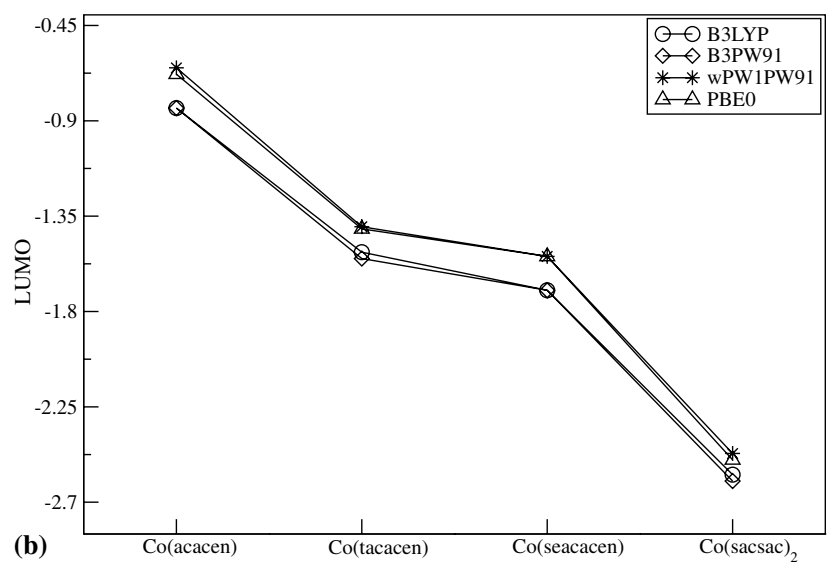

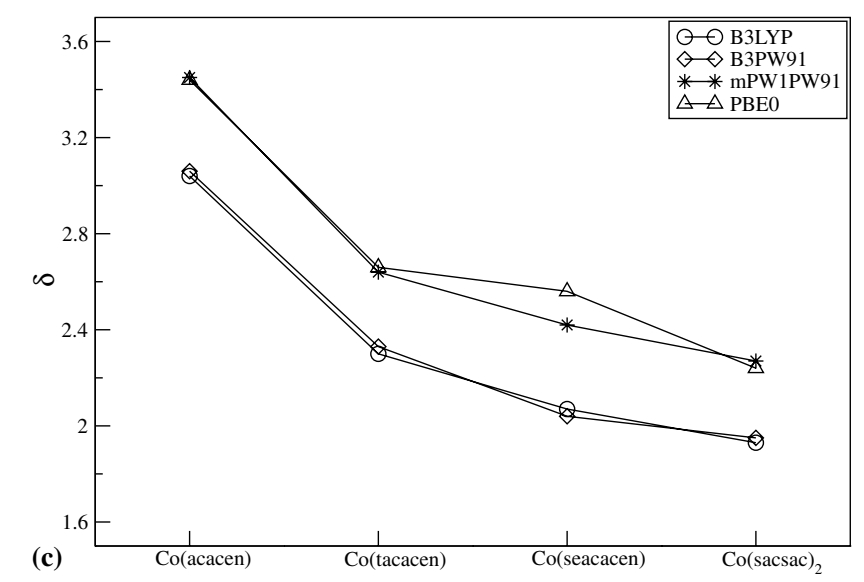

Fig. 3. Orbital energies (in eV): (a) highest occupied (HOMO); (b) lowest unoccupied (LUMO) molecular orbitals; and (c) the HOMO-LUMO gap $\delta$. 
electronic energy levels of the five d-orbitals is directly responsible of the anisotropy of the g-values.

\subsection{The A-tensor}

Unpaired electrons interact with external magnetic fields. This arise from the Zeeman effect and from the hyperfine coupling with nuclei having non-zero spins. For a nucleus $\mathrm{N}$ located at $\mathbf{r}_{\mathrm{N}}$, the isotropic (Fermi-contact) component of the hyperfine interaction tensor, $a_{\mathrm{F}}$, is related to the local spin density through [54]

$a_{\mathrm{F}}(N)=\frac{8 \pi}{3} \beta_{\mathrm{e}} \beta_{\mathrm{N}} g_{\mathrm{N}} \sum_{\mu, v} \mathbf{P}_{\mu \nu}^{\alpha-\beta}\left\langle\phi_{\mu}\left|\delta\left(\mathbf{r}-\mathbf{r}_{\mathrm{N}}\right)\right| \phi_{v}\right\rangle$

where $\beta_{\mathrm{e}}, \beta_{\mathrm{N}}$, and $\mathbf{g}_{\mathrm{N}}$ are the electronic and nuclear magnetons and the nuclear magnetogyric ratio, the indices $\mu$ and $v$ run over the basis functions, $\mathbf{P}_{\mu \nu}^{\alpha-\beta}$ is the difference between the density matrices of spin $\alpha$ and spin $\beta$ electrons and $\delta\left(\mathbf{r}-\mathbf{r}_{\mathrm{N}}\right)$ is the Dirac delta. Fig. 4 represents the isotropic Fermi contact coupling values for Co(II) nuclei for the corresponding complexes, using the four models cited above. The maximal difference between these values is $10^{-3} \mathrm{~cm}^{-1}$. The determination of the spin densities (cf. Eq. (12)) depends strongly on the hybrid method employed. More precisely, the dependence is on the amount of the exact HF exchange combined to pure DFs, and how the model is built. This is related to the spin contamination [15] which can be a problem with hybrid functionals when treating TM complexes. The anisotropic hyperfine coupling tensor, can be expressed as

$$
A_{\mathrm{kl}}(N)=\beta_{\mathrm{e}} \beta_{\mathrm{N}} \mathrm{g}_{\mathrm{e}} g_{\mathrm{N}} \sum_{\mu, v} \mathbf{P}_{\mu \nu}^{\alpha-\beta}\left\langle\phi_{\mu}\left|\mathbf{r}_{\mathrm{N}}^{-\mathbf{5}}\left(\mathbf{r}_{\mathrm{N}}^{2} \delta_{\mathrm{kl}}-3 \mathbf{r}_{\mathrm{N}, \mathrm{k}} \mathbf{r}_{\mathrm{N}, 1}\right)\right| \phi_{v}\right\rangle
$$

The A-tensor is always traceless, and after diagonalization it leads to the main components: $\mathbf{A}_{x x}, \mathbf{A}_{y y}$ and $\mathbf{A}_{z z}$. Table 2 compares computed and experimental $\mathbf{A}$-tensor values at the metal nuclei for the four complexes. One have to keep in mind, however, that the experimental determination of

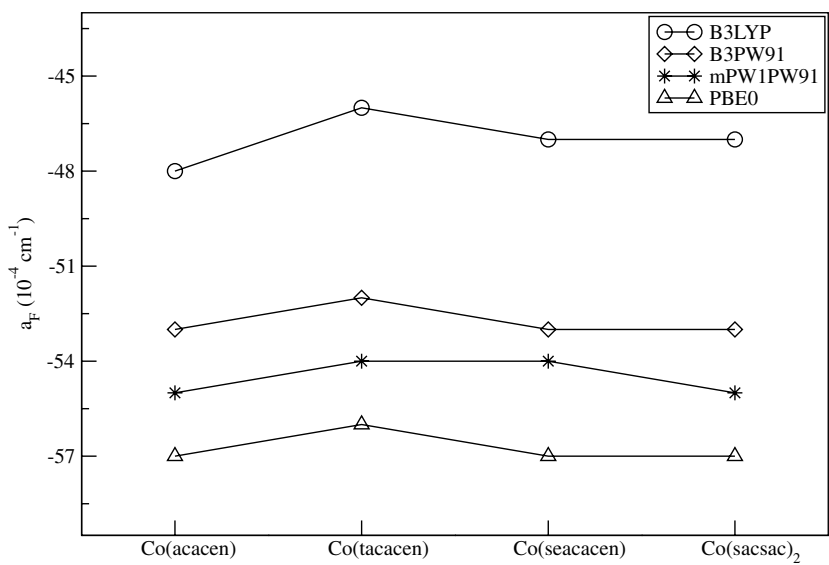

Fig. 4. The isotropic Fermi contact coupling, $a_{\mathrm{F}}$, values using different hybrid functionals.
Table 2

The calculated and the observed A-tensor values (in $10^{-4} \mathrm{~cm}^{-1}$ ) for the cobalt complexes

\begin{tabular}{llrrrr}
\hline & & Co(acacen) & Co(tacacen) & Co(seacacen) & Co(sacsac) \\
\hline B3LYP & $\mathbf{A}_{x x}$ & 120 & 114 & 112 & 110 \\
& $\mathbf{A}_{y y}$ & 37 & 36 & 33 & 32 \\
& $\mathbf{A}_{z z}$ & 42 & 37 & 40 & 35 \\
B3PW91 & $\mathbf{A}_{x x}$ & 119 & 112 & 110 & 105 \\
& $\mathbf{A}_{y y}$ & 37 & 35 & 33 & 31 \\
& $\mathbf{A}_{z z}$ & 42 & 37 & 37 & 34 \\
mPW1PW91 & $\mathbf{A}_{x x}$ & 121 & 115 & 113 & 107 \\
& $\mathbf{A}_{y y}$ & 39 & 36 & 32 & 29 \\
& $\mathbf{A}_{z z}$ & 42 & 39 & 41 & 38 \\
PBE0 & $\mathbf{A}_{x x}$ & 120 & 114 & 113 & 106 \\
& $\mathbf{A}_{y y}$ & 38 & 35 & 32 & 29 \\
& $\mathbf{A}_{z z}$ & 42 & 39 & 41 & 37 \\
Exp [18] & $\mathbf{A}_{x x}$ & 116 & 95 & 161 & 105 \\
& $\mathbf{A}_{y y}$ & 38 & 23 & 27 & 35 \\
& $\mathbf{A}_{z z}$ & 35 & 28 & 14 & 35 \\
\hline
\end{tabular}

the A-tensor is not very accurate, since more distant atoms in the solid seems to have a considerable effect on the hyperfine interaction [18]. Thus, the best choice of the functional depends strongly on the electronic structure of the TM complex $[16,15]$. While PBE0 and mPW1PW91 show better performance to calculate the $\mathrm{g}$-tensors than B3LYP and B3PW91. The four hybrid functionals perform almost equally to predict the A-tensor values. The results for co(acacen) and $\operatorname{co}(\operatorname{sacsac})_{2}$ are in a good agreement with the experiment. This is not the case for co(tacacen) and co(seacacen), where the $\mathbf{A}_{x x}$ components are not closer to the corresponding experimental values. Theoretically, correlation between the different complexes and a given hybrid method can be established. For a given hybrid functional, one can observe that the $\mathbf{A}_{x x}$ and $\mathbf{A}_{y y}$ values decrease in the following order: co(acacen) $\rightarrow$ co(tacacen $\rightarrow \operatorname{co}($ seacacen $) \rightarrow \operatorname{co}(\text { (sacsac })_{2}$. Such a trend is not observed experimentally, which confirms the above remark about the inaccuracy of the experimental determination of the A-tensor. Even if the anisotropy of the A-values is correctly reproduced, however, the computed values are of moderate quality $[5,15]$. There are various reasons why the hyperfine coupling of TM complexes presents such a difficulties. Among them, the core-shell spin polarization, which is mainly due to exchange interactions between singly occupied (SOMO) metal 3d orbitals, and the outermost doubly occupied $3 \mathrm{~s}$ - and $2 \mathrm{~s}$-type core shells. This interaction is still a challenge for the present-day functionals.

\section{Conclusions}

The interest of first principle calculations of experimental observables resides in the ability to link the electronic structure of a material to the experimental observable using relevant and adequate theoretical approaches. The main objective of the present study was to show the performance 
of the hybrid DFT to predict accurately the g- and A-tensors in TM systems, and to reproduce their anisotropic behavior in a planar symmetry for a low-spin $d^{7} \mathrm{Co}(\mathrm{II})$ complexes. The results were discussed mainly from a numerical point of view; i.e. in relation to the hybrid functionals used. Indeed, the topic of this work do not focus on a chemical point of view in relation to the nature of the ligands involved. Different hybrid models were used, and it is proven their superiority over the pure DFs. Among these hybrid methods, the PBE0 functional provides most accurate values, especially for the g-tensor. mPW1PW91 performs also efficiently. However, some problems remain to be investigated concerning the calculation of the hyperfine interaction constants (the A-tensor) of inorganic systems, which remains a challenge to quantum chemistry.

\section{Acknowledgement}

This work is supported by the Swiss National Science Foundation.

\section{References}

[1] J.E. Wertz, J.R. Bolton, Electron Spin Resonance, Chapman \& Hall, New York, 1986.

[2] J.E. Harriman, Theoretical Foundations of Electron Spin Resonance, Academic Press, New York, 1978.

[3] G.H. Lushington, F. Grein, J. Chem. Phys. 106 (1997) 3292.

[4] T. Ziegler, Can. J. Chem. 73 (1995) 743.

[5] M. Atanasov, E.J. Baerends, P. Baettig, R. Bruyndonckx, C. Daul, C. Rauzy, M. Zbiri, Chem. Phys. Lett. 399 (2004) 433.

[6] M. Kaupp, R. Reviakine, O.L. Malkina, A. Arbuznikov, B. Schimmelpfenning, V.G. Malkin, J. Comput. Chem. 23 (2002) 794.

[7] M. Buhl, O.L. Malkina, V.G. Malkin, Helv. Chim. Acta 79 (1996) 742.

[8] F. Neese, J. Chem. Phys. 115 (2001) 11080.

[9] E. van Lenthe, J.G. Snijders, E.J. Baerends, J. Chem. Phys. 105 (1996) 6505.

[10] E. van Lenthe, R. van Leeuwen, E.J. Baerends, J.G. Snijders, Int. J. Quantum Chem. 57 (1996) 281.

[11] A.V. Arbuznikov, M. Kaupp, V.G. Malkin, R. Reviakine, O.L. Malkina, Phys. Chem. Chem. Phys. 4 (2002) 5467.

[12] A.D. Becke, M.R. Roussel, Phys. Rev. A 39 (1989) 3761.

[13] A.D. Becke, J. Comput. Chem. 20 (1999) 63.

[14] P.R.T. Schpper, O.V. Gritsenko, S.J.A. van Gisbergen, E.J. Baerends, J. Chem. Phys. 112 (2000) 652.

[15] M. Munzarova, M. Kaupp, J. Phys. Chem. A 103 (1999) 9966.

[16] M.L. Munzarova, P. Kubacek, M. Kaupp, J. Am. Chem. Soc. 112 (2000) 11900

[17] M. Zbiri, S. Saha, C. Adhikary, S. Chaudhuri, C. Daul, S. Koner, Inorg. Chim. Acta 359 (2006) 1193.

[18] C. Daul, C.W. Schlaepfer, A. von Zelewsky, Struct. Bond. 36 (1978) 128.

[19] J. Harris, Phys. Rev. B 29 (1984) 1648.

[20] A.D. Becke, J. Chem. Phys. 88 (1988) 1053.

[21] A.D. Becke, Phys. Rev. A 38 (1988) 3098.

[25] C. Lee, W. Yang, R.G. Parr, Phys. Rev. B 37 (1988) 785.
[26] P.J. Stephens, F.J. Devlin, C.F. Chabalowski, M.J. Frisch, J. Phys. Chem. 98 (1994) 11623.

[28] J.P. Perdew, M. Ernzerhof, A.D. Burke, J. Chem. Phys. 105 (1996) 9982.

[29] A.D. Burke, M. Ernzerhof, J.P. Perdew, Chem. Phys. Lett. 265 (1997) 115

[30] J.P. Perdew, K. Burke, M. Ernzerhof, Phys. Rev. Lett. 77 (1996) 3865.

[31] J.P. Perdew, K. Burke, M. Ernzerhof, Phys. Rev. Lett. 78 (1997) 1396 (Erratum).

[32] J.A. Pople, M. Head-Gordon, D.J. Fox, K. Raghavachari, L.A. Curtiss, J. Chem. Phys. 90 (1989) 5622.

[33] M.J. Frisch, G.W. Trucks, H.B. Schlegel, G.E. Scuseria, M.A. Robb, J.R. Cheeseman, J.A. Montgomery Jr., T. Vreven, K.N. Kudin, J.C. Burant, J.M. Millam, S.S. Iyengar, J. Tomasi, V. Barone, B. Mennucci, M. Cossi, G. Scalmani, N. Rega, G.A. Petersson, H. Nakatsuji, M. Hada, M. Ehara, K. Toyota, R. Fukuda, J. Hasegawa, M. Ishida, T. Nakajima, Y. Honda, O. Kitao, H. Nakai, M. Klene, X. Li, J.E. Knox, H.P. Hratchian, J.B. Cross, V. Bakken, C. Adamo, J. Jaramillo, R. Gomperts, R.E. Stratmann, O. Yazyev, A.J. Austin, R. Cammi, C. Pomelli, J.W. Ochterski, P.Y. Ayala, K. Morokuma, G.A. Voth, P. Salvador, J.J. Dannenberg, V.G. Zakrzewski, S. Dapprich, A.D. Daniels, M.C. Strain, O. Farkas, D.K. Malick, A.D. Rabuck, K. Raghavachari, J.B. Foresman, J.V. Ortiz, Q. Cui, A.G. Baboul, S. Clifford, J. Cioslowski, B.B. Stefanov, G. Liu, A. Liashenko, P. Piskorz, I. Komaromi, R.L. Martin, D.J. Fox, T. Keith, M.A. Al-Laham, C.Y. Peng, A. Nanayakkara, M. Challacombe, P.M.W. Gill, B. Johnson, W. Chen, M.W. Wong, C. Gonzalez, J.A. Pople, Gaussian 03, Revision C.02, Gaussian Inc., Wallingford, CT, 2004.

[34] A.D. Becke, J. Chem. Phys. 98 (1993) 5648.

[35] C. Lee, W. Yang, R.G. Parr, Phys. Rev. B 37 (1988) 785.

[36] B. Miehlich, A. Savin, H. Stoll, H. Preuss, Chem. Phys. Lett. 157 (1989) 200

[37] J.P. Perdew, J.A. Chevary, S.H. Vosko, K.A. Jackson, M.R Pederson, D.J. Singh, C. Fiolhais, Phys. Rev. B 46 (1992) 6671.

[38] J.P. Perdew, K. Burke, Y. Wang, Phys. Rev. B 54 (1996) 16533.

[39] C. Adamo, V. Barone, J. Chem. Phys. 108 (1998) 646.

[40] C. Adamo, V. Barone, J. Chem. Phys. 110 (1999) 6158.

[41] C. Adamo, G.E. Scuseria, V. Barone, J. Chem. Phys. 111 (1999) 2889.

[42] J.L. Dodds, R. McWeeny, A.J. Sadlej, Mol. Phys. 41 (1980) 1419.

[43] K. Wolinski, J.F. Hilton, P. Pulay, J. Am. Chem. Soc. 112 (1990) 8251.

[44] F. Cariati, F. Morazzoni, C. Busetto, G. Del Piero, A. Zazetta, J. Chem. Soc., Dalton Trans. 4 (1976) 342.

[45] V. Barone, in: D.P. Chong (Ed.), In Recent Advances in Density Functional Methods. Part I, World Scientific Publ. Co., Singapore, 1996.

[46] V.A. Rassolov, M.A. Ratner, J.A. Pople, P.C. Redfern, L.A. Curtiss, J. Comput. Chem. 22 (2001) 976.

[47] G.A. Petersson, M.A. Al-Laham, J. Chem. Phys. 94 (1991) 6081.

[48] K. Raghavachari, G.W. Trucks, J. Chem. Phys. 91 (1989) 1062.

[49] O.L. Malkina, J. Vaara, B. Schimmelpfenning, M. Munzarova, V.G. Malkin, M. Kaupp, J. Am. Chem. Soc. 122 (2000) 9206.

[50] G.H. Lushington, F. Grein, Theor. Chim. Acta 93 (1996) 259.

[51] M. Engstrom, B. Minaev, O. Vahtras, H. Agren, Chem. Phys. 237 (1998) 149.

[52] G. Schreckenbach, T. Ziegler, J. Phys. Chem. A 101 (1997) 3388.

[53] F. Neese, J. Chem. Phys. 122 (2005) 34107.

[54] W. Weltner, Magnetic Atoms and Molecules, van Nostrand, New York, 1983. 